\title{
Lattice QCD with Exponentially Small Chirality Breaking.
}

\author{
A.A.Slavnov * \\ Center for Computational Physics, University of Tsukuba, \\ Tsukuba, Ibaraki 305, Japan
}

October 16, 2018

\begin{abstract}
A new multifermion formulation of lattice QCD is proposed. The model is free of spectrum doubling and preserves all nonanomalous chiral symmetries up to exponentially small corrections. It is argued that a small number of fermion fields may provide a good approximation making computer simulations feasible.
\end{abstract}

\section{Introduction}

It was believed for a long time that any local regularization of a theory which posesses a chiral symmetry breaks a manifest chiral invariance. In the context of lattice models this statement is the essence of the Nielsen-Ninomiya "no-go" theorem [1]. However in our paper [2] it was shown that anomaly free models, such as the Standard Model, do allow a local chiral invariant regularization if one introduces the regularized action containing infinite number of fermion fields. Application of this idea to lattice theories lead to the models which have no spectrum doubling and posess exact chiral invariance in the continuum limit [3], [4]. The method was also checked by nonperturbative simulations of some $2 d$ models [5]. R.Narayanan and H.Neuberger showed [6] that this approach is closely related to the five dimensional domain wall model proposed by D.Kaplan [7]. Introduction of an infinite series of fermion fields may be considered as a discretization of the fifth dimension.

Although originally it might seem that considering an infinite series of fermions is too high price, by now it is widely accepted that it is the only way to preserve the exact chiral symmetry without tuning additional parameters. Many present

*on leave from Steklov Mathematical Institute, Gubkina st. 8, GSP-1, 117966 Moscow, Russia 
approaches like overlapp formalism [8] or different versions of domain wall fermions [9], [10] use this idea.

The main question is whether these approaches allow efficient nonperturbative calculations. Considering realistic chiral gauge models on the lattice seems at present to be too hard for computer simulations. On the top of the usual problem of simulating fermion determinants there is an additional difficulty, as a chiral determinant being complex does not allow straightforward Monte-Carlo simulations. So it seems reasonable to start with the study of vectorial models like QCD and to try to deal with the breaking of the global chiral symmetry.

A naive introduction of the Wilson term in QCD to remove the degeneracy of fermion spectrum breaks global chiral invariance of the action leading to appearance of quark mass counterterms as well as order $a$ chirality breaking corrections. Apart from esthetical objections it raises practical questions for numerical simulations. Whereas the Abelian chiral symmetry is indeed broken by the triangle anomaly, the $S U(2)$ chiral invariance is not affected by anomalies and is believed to be broken spontaneously. Explicit breaking of the $S U(2)$ symmetry by the Wilson term leads to a nonzero mass of $\pi$-meson which is supposed to be a pceudogoldstone boson.

To supress these effects one may try to modify the Wilson action by adding the terms killing the order a corrections [11]. It partially solves the problem, but the modified action makes computer simulations more difficult. Extensive study of this approach was carried out in the papers (see [12] and references within).

It seems preferrable to use some approach which preserves, at least approximately, the global chiral symmetry of the theory. One possibility, which is discussed now, is to use the actions, satisfying the Ginzparg-Wilson relation [13]. It was shown, that if the model satisfies this relation, a number of results which one expects in a chiral invariant theory follows [14], [15]. Moreover in this case some modified chiral symmetry is present [16]. Examples of such actions were given in the papers [17], [16. They are typically nonlinear and formally nonlocal functionals, but nonlocal effects are damped exponentially. Some qualitative confirmation of the relevance of this approach for low energy QCD was presented in the paper [18].

The multifermion models like truncated overlapp [19] or domain wall fermions [10] were also applied to the study of QCD and first numerical simulations [20], 21] gave promising results. The chirality breaking effects, in particular fermion mass renormalization, were also discussed [22], [23].

In the present paper I propose a new version of a multifermion formulation of QCD. The model has no species doubling, does not require any fine tuning to calculate arbitrary gauge invariant amplitudes and does not contain any dimensional parameter except for a lattice spacing. For a finite lattice spacing all chirality breaking effects are supressed exponentially. Although the model includes an infinite number of fermion species, the convergence of the corresponding series is very fast, and one can hope that it may be truncated by a small finite number of terms.

The paper is organized as follows. In the Section 1 we formulate a general idea of the method and prove that our effective action produces the fermion determinant differing from the gauge invariant QCD determinant by exponentially small terms. In the second section we study the chiral property of the model and demonstrate 
the absence of a perturbative quark mass renormalization as well as exponential supression of all chirality breaking terms. In the Section 3 it is shown that all nonanomalous chiral currents are conserved whereas the usual Abelian anomaly is present. In Section 4 we discuss possible applications of the model to numerical simulations.

\section{Representation of the QCD quark determinant in the multifermion model.}

To give an idea of the method we start with the continuum model describing a gauge invariant interaction of two generations of spinor fields $\chi_{n}, \quad n=1 \ldots \infty$ with the masses proportional to the number $n, m_{n}=m n$. The following equality holds

$$
\begin{gathered}
\int \exp \left\{-\sum_{k=1,2} \sum_{n=1}^{\infty} \int \bar{\chi}_{n}^{k}(x)(\hat{D}+m n) \chi_{n}^{k}(x) d x\right\} d \bar{\chi}_{n}^{k} d \chi_{n}^{k}= \\
\int \exp \left\{-\sum_{n=1}^{\infty} \int \bar{\chi}_{n}(x)\left(-D^{2}+m^{2} n^{2}\right) \chi_{n}(x) d x\right\} d \bar{\chi}_{n} d \chi_{n}= \\
C \prod_{i} \operatorname{coth}\left(\frac{\pi D_{i}}{2 m}\right) D_{i}
\end{gathered}
$$

Here $\chi_{n}$ are spinor fields with the Grassmanian parity $(-1)^{n}, D$ is a QCD Dirac operator and $D_{i}$ are it's eigenvalues. $C$ is a field independent constant.

To derive this equation we note that the integral over $\chi$ is equal to

$$
I=\prod_{n=1}^{\infty} \operatorname{det}\left(-D^{2}+m^{2} n^{2}\right)^{(-1)^{n+1}}
$$

This equation may be rewritten as follows

$$
I=\prod_{i} \exp \left\{\sum_{n=1}^{\infty} \ln \left(D_{i}^{2}+m^{2} n^{2}\right)(-1)^{n+1}\right\}
$$

Summation in the exponent (3) may be done explicitely, giving the result

$$
\sum_{n=1}^{\infty} \ln \left(D_{i}^{2}+m^{2} n^{2}\right)(-1)^{n+1}=\ln \left(\operatorname{coth} \frac{\pi\left|D_{i}\right|}{2 m}\right)+\ln \left|D_{i}\right|
$$

This proves the eq.( 1).

It follows from the eq.(1) that up to a nonessential constant factor

$$
\lim _{m \rightarrow 0} I=\operatorname{det}(D)
$$

and

$$
\lim _{m \rightarrow \infty} I=1
$$


These equations strongly suggest that if we apply analogous procedure to lattice QCD, and instead of the mass term introduce the Wilson term $W$, we get a theory which for small values of $W$ produces the QCD massless quark determinant and for large values of $W$ (doublers region) is trivial. As follows from eq. (44) the doublers contribution is supressed by the factor $\exp \left\{-\frac{\pi D}{2 m}\right\}$, so we expect that all chirality breaking effects are also supressed exponentially. Below we justify this conjecture.

Note that in distinction of our previous approach [3], [4] as well as different versions of domain wall fermions, which include heavy states in the domain $p \sim 0$, in the present version only massless states are present in this region, and heavy states are confined to the doublers region $p \sim \pi a^{-1}$. No additional mass parameters appear in the theory.

Our claim is that the QCD determinant may be presented as the path integral of the exponent of the following action:

$$
\begin{gathered}
I=\sum_{x, \mu} \sum_{n=-\infty, n \neq 0}^{\infty}-\bar{\psi}^{n}(x) \frac{1}{2}\left[\gamma_{\mu}\left(D_{\mu}+D_{\mu}^{*}\right)-n \kappa a D_{\mu}^{*} D_{\mu}\right] \psi_{n}(x) \\
D_{\mu}=\frac{1}{a}\left[U_{\mu}(x) \psi\left(x+a_{\mu}\right)-\psi(x)\right]
\end{gathered}
$$

Here the first term represents the chiral invariant part of the action and the second term is the Wilson term multiplied by $n$. In this equation $\kappa$ is a dimensionless parameter, which we choose in the interval $0<\kappa<1$. The fields $\psi^{n}$ have a Grassmanian parity $(-1)^{n}$.

In distinction of the eq.(1) where we considered two generations of $\chi_{n}^{k}$ fields $(k=1,2)$, and $1 \leq n \leq \infty$, in eq. ( 7) we introduced one generation, but allow $n$ to change in the interval $-\infty \leq n \leq \infty, \quad n \neq 0$. For a zero bare quark mass it makes no difference, but, as it was pointed out to me by A.Ukawa, for nonzero quark mass the choice $-\infty \leq n \leq \infty, \quad n \neq 0$ is more convinient.

Let us consider a fermion loop diagram with $L$ vertices, which may be presented by the following integral

$$
\begin{gathered}
\Pi_{L}\left(q_{1} \ldots q_{L-1}\right)=\int_{-\pi a^{-1}}^{\pi a^{-1}} d^{4} p \sum_{n=-\infty, n \neq 0}^{\infty}(-1)^{n} \operatorname{Tr}\left[G(p) \Gamma_{i}\left(p, q_{1}\right) G\left(p+q_{1}\right) \Gamma_{j}\left(p, q_{2}\right) \ldots\right]= \\
=2 \int_{-\pi a^{-1}}^{\pi a^{-1}} \sum_{n=1}^{\infty}(-1)^{n} \frac{F_{0}(p, q)+n^{2} F_{1}(p, q)+\ldots}{\left(s^{2}+m^{2} n^{2}\right)\left(s_{1}^{2}+m_{1}^{2} n^{2}\right) \ldots}
\end{gathered}
$$

Here $\Gamma_{i}$ denotes the interaction vertices and $q_{l}$ is a total momentum entering the corresponding vertex. The fermion propagators $G(p)$ look as follows

$$
\begin{gathered}
G_{n}(p)=\frac{i \hat{s}+m n}{s^{2}+m^{2} n^{2}} \\
s_{\mu}^{l}=a^{-1} \sin \left[\left(p+q^{1}+\ldots+q^{l}\right)_{\mu} a\right] \\
m^{l}=\kappa a^{-1} \sum_{\mu}\left(1-\cos \left(p+q^{1}+\ldots+q^{l}\right)_{\mu}\right)
\end{gathered}
$$


The eq.(9) may be rewritten in the following form

$$
\Pi_{L}=2 \int_{-\pi a^{-1}}^{\pi a^{-1}} d^{4} p \sum_{l=0}^{L-1} \sum_{n=1}^{\infty}(-1)^{n} \frac{A_{l}(p, q)}{s_{l}^{2}+m_{l}^{2} n^{2}}
$$

Here $A_{l}$ are some functions of $p, q$, which do not depend on $n$. They satisfy the equations

$$
\begin{gathered}
\sum_{l} A_{l} \prod_{i=0, i \neq l}^{L-1} s_{i}^{2}=F_{0}(p, q) \\
\ldots \\
\sum_{l} A_{l} \prod_{i \neq l} m_{i}^{2}=0
\end{gathered}
$$

The summation over $n$ can be done as before, giving the result

$$
\Pi_{L}=\int_{-\pi a^{-1}}^{\pi a^{-1}} d^{4} p\left[-\sum_{l=0}^{L-1} \frac{\pi A_{l}(p, q)}{m_{l} \sqrt{s_{l}^{2}} \sinh \left(\pi \sqrt{s_{l}^{2}} m_{l}^{-1}\right)}+\frac{A_{l}(p, q)}{s_{l}^{2}}\right]
$$

One sees that for small $p+q_{1}+\ldots+q_{l}$ the first term is damped exponentially and the second one describes the amplitude for massless quarks. For $p+q_{1}+\ldots+q_{l} \sim \pi a^{-1}$ the first term is

$$
\sim-\frac{A_{l}(p, q)}{s_{l}^{2}}
$$

and exactly compensates the second one providing the doublers supression.

Now we shall study eqs. (9- 15) in more details and prove that the amplitudes (9) coincide with the gauge invariant QCD amplitudes up to the terms which decrease exponentially when $a \rightarrow 0$.

Firstly we consider the case when all momenta $q_{l}$ are external. Then we can assume that

$$
\left|q_{l}\right|<\epsilon a^{-1}, \quad \epsilon<1
$$

The integration domain in the eq.(9) can be separated into three parts:

$$
\begin{gathered}
V_{1}:|p|<\epsilon a^{-1} ; \quad V_{2}: L \epsilon a^{-1} \leq|p| \leq(\pi-L \epsilon) a^{-1} \\
V_{3}:(\pi-L \epsilon) a^{-1} \leq|p| \leq \pi a^{-1}
\end{gathered}
$$

In the region $V_{2}$ the function

$$
\left.s_{l}^{-2} \simeq\left(\sum_{\mu} \sin \left(p_{\mu} a\right)\left(1+\cot \left(p_{\mu} a\right)\left(q_{1}+\ldots+q_{l}\right) a\right)\right)\right)^{-2}
$$

may be expanded in terms of $q_{l} a$. It is easy to see that the the functions $A_{l}$ also may be expanded in terms of $q_{l} a$. Therefore integrating the second term in the eq.(15) over $p$ one gets a local polynomial in $q_{l}$. Analogous reasonings show that the first term in the eq.(15) also contributes a local polynomial in $q_{l}$.

In the domain $V_{3}$ we cannot use the expansion (19) as in this region $\sin \left(p_{\mu} a\right) \sim 0$. However the complete r.h.s. of the eq.(15) still allows the expansion in terms of $q_{l} a$. 
Indeed, in this region $s_{l}^{2}<m_{l}^{2}$, and the r.h.s. of the eq(15) may be presented as follows

$$
\Pi_{L}=\int_{-\pi a^{-1}}^{\pi a^{-1}} d^{4} p \sum_{l=0}^{L-1} A_{l}\left\{\frac{1}{s_{l}^{2}}-\frac{1}{s_{l}^{2}}\left(1-\frac{\pi^{2} s_{l}^{2}}{6 \pi^{2} m_{l}^{2}}+O\left(\frac{s^{4}}{m^{4}}\right)\right)\right\}
$$

The terms $\sim s_{l}^{-2}$ cancel and the remaining terms may be expanded in terms of $q_{l} a$.

In the region $V_{1}$ we use the representation (15) for the amplitude (9). In this region $\left|a\left(p-q_{l}\right)\right|<2 \epsilon$ and therefore

$$
\sum_{\mu} \sin ^{2}\left[a\left(p-q_{l}\right)_{\mu}\right]>\left[\sum_{\mu} 1-\cos \left(p-q_{l}\right)_{\mu} a\right]^{2}
$$

Hence the first term in the eq.(15) is supressed exponentially by the factor

$$
\frac{1}{\sinh \left(\pi \sqrt{s_{l}^{2}} m_{l}^{-1}\right)} \sim \exp \left\{-\frac{\pi}{\epsilon \kappa}\right\}
$$

The second term gives

$$
\int_{-\epsilon a^{-1}}^{\epsilon a^{-1}} d^{4} p \sum_{l=0}^{L-1} \frac{A_{l}(p, q)}{s_{l}^{2}}=\int_{\epsilon a^{-1}}^{\epsilon a^{-1}} d^{4} p \frac{F_{0}(p, q)}{s_{0}^{2} \ldots s_{L-1}^{2}}
$$

where the eq.(14) was used. This is exactly the expression which one would get in the chiral invariant theory with no Wilson term. In the limit $a \rightarrow 0$ this term reproduces the diagrams of the continuum QCD. It is worthwhile to notice that if only this term were present, the gauge invariance could be broken, as cutting the Brillouin zone is not a gauge invariant procedure. In our construction the gauge invariance is preserved at any stage of calculations. Possible noninvariant terms arising from cutting the Brillouin zone in the eq.(23) are compensated by local counterterms generated by the integral over $V_{2}, V_{3}$. It would be wrong just to drop the integration over $V_{2}, V_{3}$. The local counterterms coming from these integrals are crucial for maintaining the gauge invariance of the amplitudes. So we proved that for one loop diagrams, when all $q_{l}$ are external momenta, $q_{l}<\epsilon a^{-1}$, the amplitude (9) differs from the gauge invariant QCD amplitude by exponentially small terms. The value of the correction term for the fixed $a$ depends on external momenta $q$. In particular for $q=0$ it is zero for any $a$. The rate of the exponential damping depends also on the parameter $\kappa$, and one may have a temptation to take $\kappa$ very close to zero. It is clear however that if one puts $\kappa=0$, the correction term indeed disappear, but the doubler states reappear. To determine possible values of $\kappa$ we note that considering the contribution of the region $V_{3}$ we used the expansion in terms of $q a$. It is easy to see that for $p \sim \pi a^{-1}$ the effective expansion parameter is $\frac{q a}{\kappa}$. So the expansion to make sense we need $\kappa>q a$. In particular we may take $\kappa=\epsilon$.

In the next section we consider the multiloop diagrams and prove that they also reproduce chiral invariant QCD amplitudes up to exponentially small corrections. To do that we have to study more closely the global chiral invariance of our model. 


\section{Global chiral invariance of the model.}

In this section we study the invariance of our model under global chiral transformations. We show, that as expected in a chiral invariant theory, a perturbative quark mass renormalization is absent. All chirality breaking effects are supressed exponentially. The divergency of any amplitude including an $S U(2)$ chiral current vertex, or any other chiral current, corresponding to an anomaly free group is zero up to exponentially small corrections. At the same time the divergency of the Abelian axial current is not zero, producing the usual chiral anomaly.

To give an idea of the mechanism which provides the supression of chirality breaking effects we firstly consider a simplified case. All chirality breaking effects arise due to the presence of the Wilson term and therefore vanish when $n=0$. Let us suppose that some chirality breaking term, for example the fermion mass renormalization, is simply proportional to $n$ : $\delta m_{n}=g^{2} M(g) n$.

Introducing such counterterm is equvalent to shifting the Wilson term by $g^{2} M n$. To get the expression for the QCD determinant we still may use the eq.s( 9- 15), changing everywhere $m_{l}$ by $m_{l}+g^{2} M$. It is sufficient to consider the case when $\left|q_{l}\right|<\epsilon a^{-1}$ and the integration domain in the eq.( 15) is restricted to $\left(-\epsilon a^{-1}, \epsilon a^{-1}\right)$, as it was shown above that larger values of $p$ produce only local counterterms needed to maintain the gauge invariance. The expression for the QCD amplitude acquires the form:

$$
\begin{gathered}
\Pi_{L}=\int_{-\epsilon a^{-1}}^{\epsilon a^{-1}} d^{4} p\left[\frac{A_{l}(p, q)}{s_{l}^{2}}-\right. \\
\left.-\sum_{l=0}^{L-1} \frac{\pi A_{l}(p, q)}{\left(m_{l}+g^{2} M\right) \sqrt{s_{l}^{2}} \sinh \left(\pi \sqrt{s_{l}^{2}}\left(m_{l}+g^{2} M\right)^{-1}\right)}\right]
\end{gathered}
$$

The sum of the first terms in the eq.(24) due to eq.(14) is equal to

$$
\int_{-\epsilon a^{-1}}^{\epsilon a^{-1}} d^{4} p \frac{F_{0}(p, q)}{s_{0}^{2} \ldots s_{L-1}^{2}}
$$

which coincides with the result obtained before for the model without mass counterterm insertions. Expanding the second term into Taylor series over $g$ one sees that all the coefficients are exponentially damped, being proportional to $\exp \left\{-\pi \sqrt{s_{l}^{2}} m_{l}^{-1}\right\} \leq$ $\exp \left\{-\pi(\kappa \epsilon)^{-1}\right\}$. So for the simplified case we proved our statement.

However in general chirality breaking terms may have more complicated dependence on $n$. Below we shall show that our statement remains true in a general case as well, although the proof is more involved.

To prove the chiral invariance of the determinant it is sufficient to show that it's derivative with respect to $\kappa$ is proportional to some chiral invariant amplitude. Note that this derivative is not necessarily zero as chiral invariant counterterms may also depend on $\kappa$. We demonstrate that it is indeed true up to exponentially small terms.

We study the following object

$$
I=\frac{\partial}{\partial \kappa}\left(\sum_{n=-\infty, n \neq 0}^{\infty}(-1)^{n} \operatorname{Tr} \ln D_{n}\right)=
$$




$$
=\sum_{n}(-1)^{n} \operatorname{Tr}\left(n W(D+n \kappa W)^{-1}\right)
$$

Here $W$ is the usual Wilson term. This equation may be rewritten as follows

$$
I=\sum_{n}(-1)^{n} \operatorname{Tr}\left(n W G_{n}\right)
$$

wher $G_{n}$ is the Green function of the Wilson-Dirac operator in the external gauge field. This function satisfies the chiral Ward identities which in the continuum notations may be written as follows

$$
\begin{gathered}
G_{n}(x, y) \gamma_{5}+\gamma_{5} G_{n}(x, y)=2 i n \kappa \int G_{n}(x, z) \gamma_{5} W(z) G_{n}(z, y) d z \\
\gamma_{5} G_{n} \gamma_{5}=-G_{-n}
\end{gathered}
$$

The Green function $G_{n}$ may be presented as a sum $G_{n}=G_{n}^{s}+G_{n}^{b}$ where $G_{n}^{s}$ is a chiral symmetric part which anticommutes with $\gamma_{5}$ and is even in $n$. $G_{n}^{b}$ is a chirality breaking part which commutes with $\gamma_{5}$ and is odd in $n$. It follows from eq(28) that

$$
G_{n}^{b}(x, y)=i n \kappa \gamma_{5} \int G_{n}(x, z) \gamma_{5} W(z) G_{n}(z, y) d z
$$

Using these properties of $G_{n}$ one can rewrite the eq(27) in the form

$$
I=\sum_{n=-\infty, n \neq 0}^{\infty}(-1)^{n} n^{2} i \kappa \int \operatorname{Tr}\left(W(x) \gamma_{5} G_{n}(x, z) \gamma_{5} W(z) G_{n}(z, x)\right) d x d z
$$

The eq(31) is written for one loop diagrams. To get the multiloop diagrams one has to integrate it with the exponent of the gluon action. In terms of Feynman diagrams it is represented by the fermion loops with two insertions of the Wilson term $W$.

We shall start with the diagrams including one fermion loop and arbitrary number of gluon vertices and lines.

The analytic expression corresponding to the eq(31) for such diagrams may be written as follows

$$
I_{L}=\int \sum_{n=-\infty, n \neq 0}^{\infty}(-1)^{n} n^{2} \frac{\left(K_{0}+n^{2} K_{1}+\ldots\right) F\left(q_{r} \ldots q_{L-1}\right) d p d q_{r} d q_{L-1}}{\left(s_{0}^{2}+m_{0}^{2} n^{2}\right) \ldots\left(s_{L-1}^{2}+m_{L-1}^{2} n^{2}\right)}
$$

Here we wrote the expression for the diagram with $L$ quark- gluon vertices. The notations are as follows:

$$
\begin{gathered}
s_{0}=\sqrt{\left(\sum_{\mu} s_{\mu}^{2}(p)\right),} \quad m_{0}=m(p), \\
s_{l}=\sqrt{\left(\sum_{\mu} s_{\mu}^{2}\left(p+q_{1}+\ldots+q_{l}\right)\right),} \quad m_{l}=m\left(p++q_{1}+\ldots+q_{l}\right)
\end{gathered}
$$

The momenta $q_{1}, \ldots q_{r}$ are external and satisfy the condition $\left|q_{l} a\right|<\epsilon$. The momenta $q_{r+1}, \ldots q_{L-1}$ correspond to virtual gluons and may acquire arbitrary values 
in the interval $\left(-\frac{\pi}{a}, \frac{\pi}{a}\right)$. The function $F\left(q_{r}, \ldots q_{L-1}\right)$ depends only on virtual gluon momenta corresponding to gluon propagators and vertices.

We use the following decomposition of the integrand

$$
\begin{gathered}
\frac{K_{0}+n^{2} K_{1}+\ldots}{\left(s_{0}^{2}+m_{0}^{2} n^{2}\right) \ldots\left(s_{r}^{2}+m_{r}^{2} n^{2}\right)}=\sum_{l=0}^{r} \frac{B_{l}}{s_{l}^{2}+m_{l}^{2} n^{2}} \\
\frac{1}{\left(s_{r+1}^{2}+m_{r+1}^{2} n^{2}\right) \ldots\left(s_{L-1}^{2}+m_{L-1}^{2} n^{2}\right)}=\sum_{k=r+1}^{L-1} \frac{A_{k}}{s_{k}^{2}+m_{k}^{2} n^{2}}
\end{gathered}
$$

Here $B_{l}$ and $A_{k}$ are $n$-independent functions satisfying the relations

$$
\begin{aligned}
& \sum_{l} B_{l} \prod_{i \neq l} s_{i}^{2}=K_{0}, \quad \cdots \quad \sum_{l} B_{l} \prod_{i \neq l} m_{i}^{2}=0 \\
& \sum_{k} A_{k} \prod_{i \neq k} s_{i}^{2}=1, \quad \cdots \quad \sum_{k} A_{k} \prod_{i \neq k} m_{k}^{2}=0
\end{aligned}
$$

We assume that all momenta $s_{l}$ are different. It is not true for the diagrams with self energy insertions, but it will be clear from the final result that our conclusions are valid for this case as well.

Using these decompositions we may rewrite the integrand in eq(32) in the form

$$
\begin{aligned}
\tilde{I}_{L} & =\sum_{n=-\infty, n \neq 1}^{\infty}(-1)^{n} n^{2}\left(\sum_{l=0}^{r} \frac{B_{l}}{s_{l}^{2}+m_{l}^{2} n^{2}}\right)\left(\sum_{k=r+1}^{L-1} \frac{A_{k}}{s_{k}^{2}+m_{k}^{2} n^{2}}\right)= \\
& =\sum_{n}(-1)^{n} \sum_{l, k} \frac{B_{l} A_{k}}{s_{k}^{2} m_{l}^{2}-s_{l}^{2} m_{k}^{2}}\left(\frac{m_{l}^{2} n^{2}}{s_{l}^{2}+m_{l}^{2} n^{2}}-\frac{m_{k}^{2} n^{2}}{s_{k}^{2}+m_{k}^{2} n^{2}}\right)
\end{aligned}
$$

Performing the summation over $n$ one gets

$$
\tilde{I}_{L}=\sum_{l, k} \frac{B_{l} A_{k}}{s_{k}^{2} m_{l}^{2}-s_{l}^{2} m_{k}^{2}}\left[\frac{s_{l}^{2} \pi}{s_{l} m_{l} \sinh \left(\pi s_{l} m_{l}^{-1}\right)}-\frac{s_{k}^{2} \pi}{s_{k} m_{k} \sinh \left(\pi s_{k} m_{k}^{-1}\right)}\right]
$$

All the momenta $s_{l}, \quad 0 \geq l \geq r$ are small and therefore the first term gives exponentially small corrections. If some of the momenta $s_{k}$ are also small, the corresponding term is also supressed exponentially. So it is sufficient to consider the eq(39) when all $s_{k}$ are big: $\epsilon \leq\left|a\left(p+\ldots q_{k}\right)\right| \leq \pi$.

The remaining terms may be expanded in terms of $m_{l}^{2} s_{l}^{-2}$. (We recall that $q_{l} a<\epsilon$ and therefore $m_{l} \ll s_{l}$.

$$
\tilde{I}_{L}=\sum_{k=r+1}^{L-1} \frac{A_{k} s_{k}^{2} \pi}{s_{k} m_{k}^{3} \sinh \left(\pi s_{k} m_{k}^{-1}\right)} \sum_{l=0}^{r} \frac{B_{l}}{s_{l}^{2}}\left(1+\frac{m_{l}^{2} s_{k}^{2}}{s_{l}^{2} m_{k}^{2}}+\ldots\right)
$$

Let us consider the sum over $l$. Due to eq(35) the first term is equal to

$$
\sum_{l=0}^{r} \frac{B_{l}}{s_{l}^{2}}=\frac{K_{0}}{s_{0}^{2} \ldots s_{r}^{2}}=s_{r+1}^{2} \ldots s_{L-1}^{2} I_{L}^{0}
$$


where

$$
I_{L}^{0}=\frac{\operatorname{Tr}\left(\hat{s_{0}} \Gamma_{1} \hat{s_{1}} \Gamma_{2} \ldots\right)}{\prod_{l=0}^{L-1} s_{l}^{2}}
$$

is the manifestly chiral invariant amplitude corresponding to the theory without the Wilson term.

The first factor in the eq(40) may be expanded in terms of $a q_{l}$ where $q_{l}, \quad l=$ $1, \ldots r$ are "small" external momenta. Being integrated over $p, q_{r+1} \ldots q_{L-1}$ the first term in the eq(40) is equal to the manifestly chiral invariant amplitude (42) integrated with the chiral invariant function and multiplied by a polynomial of external momenta. Such a term is obviously chiral invariant.

To analyse the next terms we use the relations which follow from the formal expansion of the eq (34) in terms of $n^{2}$.

$$
\begin{gathered}
\sum_{l=0}^{r} \frac{B_{l}}{s_{l}^{2}}\left(1-n^{2} \frac{m_{l}^{2}}{s_{l}^{2}}+\ldots\right)= \\
=K_{0} \prod_{l=0}^{r} \frac{1}{s_{l}^{2}}-n^{2}\left(K_{1} \prod_{l=0}^{r} \frac{1}{s_{l}^{2}}+K_{0} \prod_{l=0}^{r} \frac{m_{l}^{2}}{s_{l}^{2}}\right)
\end{gathered}
$$

Using these relations we can rewrite the second term in the r.h.s. of the eq(40) in the form

$$
\sum_{k=r+1}^{L-1} \frac{A_{k} s_{k} \pi}{m_{k}^{3} \sinh \left(\pi s_{k} m_{k}^{-1}\right)}\left[K_{1} \prod_{l=0}^{r} \frac{1}{s_{l}^{2}}+K_{0} \prod_{l=0}^{r} \frac{m_{l}^{2}}{s_{l}^{4}}\right]
$$

The first term in brackets is equal to

$$
\frac{K_{1} \prod_{k=r+1}^{L-1} s_{k}^{2}}{K_{0}} I_{L}^{0}
$$

The factor $K_{1} K_{0}^{-1} \prod_{k} s_{k}^{2}$ may be expanded in a power series in $a q_{l}, \quad l=1, \ldots r$. Coefficients in this series are not singular at $p=0$. Therefore being integrated over $p, q_{k}, \quad k=r+1, \ldots L-1$ this term is equal to a manifestly chiral invariant amplitude $I_{0}$ multiplied by a polynomial in $q_{l}$. In the second term in the eq(44) we can expand $m_{l}^{2} s_{l}^{-4}$ in terms of $a q_{l}, \quad l=1, \ldots r$. Again it is easy to see that the coefficients of this expansion are not singular at $p=0$. Hence being integrated over $p, q_{k}$ this term produces only local counterterms.

The next order terms in the eq(40) are analyzed in the same way and lead to the same conclusion. It proves that the derivative over $\kappa$ of the diagrams under consideration is equal to a chiral invariant amplitude plus a local gauge invariant polynomial up to exponentially small corrections. Hence the QCD determinant is chiral invariant.

These reasonings are easily extended to the diagrams including more than one fermion loop. A diagram with two fermion loops may be presented as a product of two diagrams including one fermion loop integrated with some function depending on the virtual gluon momenta. For each one loop diagram one has to use the 
decompositions (34, 36). Multiplying the corresponding expressions and using the representation

$$
\frac{1}{\left(s_{l}^{2}+m_{l}^{2} n^{2}\right)\left(s_{k}^{2}+m_{k}^{2} n^{2}\right)}=\frac{1}{\left.s_{k}^{2} m_{l}^{2}-m_{l}^{2} s_{k}^{2}\right)}\left(\frac{m_{l}^{2}}{s_{l}^{2}+m_{l}^{2} n^{2}}-\frac{m_{k}^{2}}{s_{k}^{2}+m_{k}^{2} n^{2}}\right)
$$

one reduces the problem to the one considered above. Diagrams with arbitrary number of fermion loops are analyzed in the same way.

It completes the proof of the chiral invariance of any gauge invariant amplitude in our model.

\section{Chiral currents conservation. Anomalies.}

In this section we prove that the currents assosiated with nonanomalous global chiral symmetries are conserved up to exponentially small terms.

Let us consider the action (7) with the source term for a chiral current:

$$
I_{V}=I+\sum_{x, \mu} V_{\mu}^{b}(x) J_{\mu}^{b}(x)
$$

Here $J_{\mu}$ is the axial current which in the continuum case is equal to

$$
J_{\mu}^{b}(x)=\sum_{n=-\infty, n \neq 0}^{\infty} \bar{\psi}_{n}(x) \gamma_{5} \gamma_{\mu} T^{b} \psi_{n}(x)
$$

$T^{b}$ being the generators of the chiral symmetry group. $V_{\mu}$ is the source term. In the continuum chiral invariant theory this current is covariantly conserved

$$
\partial_{\mu} J_{\mu}^{b}+g t^{b c d} J_{\mu}^{c} V_{\mu}^{d}=0
$$

For the lattice action (47) including the Wilson term the corresponding equation is modified. Firstly, the Neuther current has a contribution from the Wilson term, and secondly, as the Wilson term breaks the chiral symmetry, the r.h.s. is nonzero. The analogue of the eq.(49) for the action (47) may be written as follows

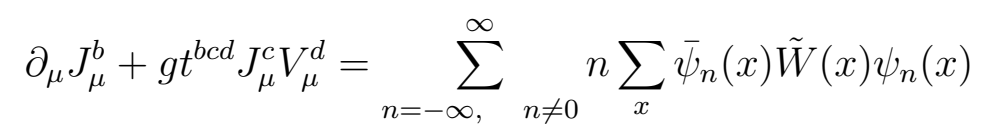

where $\partial$ is a lattice derivative. The r.h.s. of eq.(50) is due to the Wilson term and in the quantum theory is responsible for the chiral anomaly. A particular form of $\tilde{W}$ is not essential for our discussion. It is important that the r.h.s. of eq. (50) is bilinear in $\psi$ and proportional to $n$.

Being integrated with the exponent of the action (47) the eq.(50) leads to the chiral Ward identity broken by the Wilson term

$$
a^{-1} \sin \left(k_{\mu} a\right) \Gamma_{\mu \nu_{1} \ldots \nu_{n}}^{b c_{1} \ldots c_{n}}\left(k, q_{1} \ldots q_{n}\right)+
$$




$$
+g t^{b c d} \int d^{4} p \Gamma_{\mu \nu_{1} \ldots \nu_{n}}^{c c_{1} \ldots c_{n}}\left(k-p, q_{1} \ldots q_{n}\right) V_{\mu}^{d}(p)=\sum_{n=-\infty,}^{\infty} n W_{n}
$$

Here $W_{n}$ stands for the sum of diagrams generated by the r.h.s. of the eq.(50).

We shall use the differential form of this identity, which is obtained by differentiating it over $k_{\mu}$ and putting $k=0$ :

$$
\begin{gathered}
\Gamma_{\mu \nu_{1} \ldots \nu_{n}}^{b c_{1} \ldots c_{n}}\left(0, q_{1} \ldots q_{n}\right)+ \\
+\left.g t^{b c d} \int d^{4} p \partial_{\mu} \Gamma_{\mu \nu_{1} \ldots \nu_{n}}^{c c_{1} \ldots c_{n}}\left(k-p, q_{1} \ldots q_{n}\right)\right|_{k=0} V_{\mu}^{d}(p)=\left.\sum_{n=-\infty, \quad}^{\infty} n \frac{\partial W_{n}}{\partial k_{\mu}}\right|_{k=0}
\end{gathered}
$$

Here is the important difference between anomalous and nonanomalous theories. Passing from the eq. (51) to the differential identity (52) we assumed that $\frac{\partial \Gamma}{\partial k_{\mu}}$ is not singular at $k=0$. It is not true for anomalous models: the anomalous triangle diagram is singular at $k=0$. Therefore the eq.(52) is equivalent to the identity (51) only if anomaly is absent.

Now we shall show that the r.h.s. of the eq.(52) is exponentially small. To prove the absence of anomaly it is sufficient to consider one loop diagrams. The diagrams representing the r.h.s. of eq.(52) are obtained from the QCD amplitudes which we considered in the previous section by insertions of the additional vertices $\left.n \frac{\partial W}{\partial k_{\mu}}\right|_{k=0}=n \mu$, corresponding to zero external momentum. In other words they may be considered as momentum dependent counterterms which shift the Wilson term $n m \rightarrow n(m+\mu)$. The sum over $n$ gives for such diagrams the result analogous to the eq. (24)

$$
\begin{gathered}
\Pi^{L}=\int_{-\epsilon a^{-1}}^{\epsilon a^{-1}} d^{4} p\left[\frac{A_{l}(p, q)}{s_{l}^{2}}-\right. \\
\left.-\sum_{l=0}^{L-1} \frac{\pi A_{l}(p, q)}{\left(m_{l}+\mu\right) \sqrt{s_{l}^{2}} \sinh \left(\pi \sqrt{s_{l}^{2}}\left(m_{l}+\mu\right)^{-1}\right)}\right]+c . t .
\end{gathered}
$$

where c.t. stands for local counterterms. As was explained above the sum of the first term and local counterterms is equal to the gauge invariant QCD amplitude without insertions of $\mu$ and the remaining terms are proportional to $\exp \left\{-\frac{\pi s_{l}}{\mu+m_{l}}\right\}$. The diagrams with $k$ insertions of $\mu$ are given by the $k$-th term of the formal Taylor expansion of this exponent in terms of $\mu$ near the point $\mu=0$. All these terms are exponentially small. So we proved that to any nonanomalous global chiral symmetry corresponds a current which is covariantly conserved up to exponentially small corrections. At the same time the global $U(1)$ anomaly is present as it was explained before the differential Ward identity (52) does not hold in this case.

\section{Discussion}

In the previous sections we demonstrated that the lattice QCD determinant may be presented by a path integral of a local action, which is free of spectrum degeneracy and reproduces gauge invariant amplitudes of massless QCD up to exponentially 
small corrections. Nonanomalous global chiral symmetries, in particular $S U(2)$ chiral invariance are also preserved up to exponentially small corrections. No chirality breaking counterterms are needed and the model provides automatically $O(a)$ improvement.

Our procedure may be also used in the case when a nonzero bare quark mass is present. In this case one has to consider the sums of the form

$$
\sum_{n=-\infty,}^{\infty}{ }_{n \neq 0} \frac{(-1)^{n}}{s_{l}^{2}+\left(m_{l} n+m_{0}\right)^{2}}
$$

where $m_{0}$ is the bare quark mass. Representing this sum by the integral

$$
\int_{C} \frac{1}{\cos (\pi z)\left[s_{l}^{2}+\left(m_{l} z+m_{0}\right)^{2}\right]} d z
$$

where the contour $C$ encloses the real axis except for a vicinity of the point $z=0$, one sees that this integral is equal to

$$
\frac{1}{s_{l}^{2}+m_{0}^{2}}+O\left(\exp \left\{-\pi s_{l} m_{l}^{-1}\right\}\right)
$$

Of course in this case the chiral current conservation is broken by the mass term and chirality breaking corrections $\sim m_{0}$ are present.

Our effective action includes an infinite series of fields, and for practical simulations it is important to know how sensitive is the procedure to cutting this series by some finite number of terms. Fist of all we note that cutting the series does not spoil the gauge invariance of the model. So all the arguments using this invariance are still applicable. Due to the fast convergence of the series we expect that a small number of terms may provide a good approximation. This number depends on the value of external momenta $q_{l}$, which are relevant for the process under consideration. As we showed above the integration over momenta bigger than $q_{l}$ produces local counterterms. Therefore it is sufficient to study the convergence of the series for $|p|<\left|q_{l}\right|$. The parameter which determines the value of the correction term is $\sqrt{s_{l}^{2}} m_{l}^{-1}=b$. For external momenta $|q a|<1$ this parameter is $\geq 2($ for $\kappa=1)$.

The following simple observation may be useful for numerical simulations. For large values of $b$ the correction term is small, however to approximate it by a finite series one needs to cut it by some number $N \gg b$. We recall however that in our scheme the QCD determinant is presented as a product of the determinants corresponding to the fields $\psi_{n}, \quad-\infty<n<\infty, \quad n \neq 0$. To improve the convergence for large $b$ one can cut the series asymmetrically $-N \leq n \leq N+1$. Then for $b \gg N$ and even $N$ the contribution of the fields with negative $n$ is close to zero, whereas the contribution of the fields with positive $n$ produces the correct result.

Numerical estimates of the correction term for $q a<1 / 4, \quad \kappa=1 / 4$ show that cutting the series by $-2 \leq n \leq 3$ gives the relative correction $\sim 0.02$. Of course it is a rather crude and naive estimate and only real simulations may prove the efficiency of the method. However it raises a hope that relatively small amount of fields may be sufficient to have a strong supression of chirality breaking effects. We recall also 
that only half of the $\psi_{n}$ fields are fermions which is important for simulations with dynamical quarks. Having in mind that these simulations do not require a fine tuning of mass parameters and introduction of "improving" terms, one may think that this model provides a competitive method of simulations in lattice QCD.

\section{Acknowledgements.}

This work was done while the author was visiting Center for Computational Physics of the University of Tsukuba. I am grateful to Y.Iwasaki, A.Ukawa and all members of CCP for hospitality and fruitful discussions. I acknowledge stimulating discussions with Y.Kikukawa on related problems. This work is supported in part by Russian Basic Research Foundation under grant 96-01-00551, Presidential grant for the support of leading scientific schools and INTAS-96-370

\section{References}

[1] H.B.Nielsen, M.Ninomiya, Nucl.Phys. B105 (1981) 219.

[2] S.A.Frolov, A.A.Slavnov, Phys.Lett. B309 (1993) 344.

[3] S.A.Frolov, A.A.Slavnov, Nucl.Phys. B411 (1994) 647

[4] A.A.Slavnov, Phys.Lett. B348 (1993) 344.

[5] A.A.Slavnov, N.V.Zverev, Phys.Lett. B420 (1998) 323.

[6] R.Narayanan, H.Neuberger, Phys.Lett. B302 (1993) 62.

[7] D.B.Kaplan, Phys.Lett. B288 (1992) 342.

[8] R.Narayanan, H.Neuberger, Nucl.Phys. B412 (1994) 574.

[9] Y.Shamir, Nucl.Phys. B406 (1993) 90.

[10] V.Furman, Y.Shamir, Nucl.Phys. B439 (1995) 54.

[11] B.Sheikholeslami, R.Wohlert, Nucl.Phys. B259 (1985) 572.

[12] M.Lüscher, S.Sint, R.Sommer, P.Weisz, Nucl.Phys. B478 (1996) 365.

[13] P.H.Ginzparg, K.G.Wilson, Phys.Rev. D25 (1982) 2649.

[14] P.Hasenfratz, Nucl.Phys.B (Proc.Suppl) 63 (1998) 53.

[15] P.Hasenfratz, V.Laliena, F.Niedermayer, hep-lat/9801021.

[16] M.Lüscher, hep-lat/9802011.

[17] H.Neuberger, Phys.Lett. B417 (1998); hep-lat/9801031.

[18] Sh.Chandrasekharan, hep-lat/9805015. 
[19] H.Neuberger, Phys.Rev. D57 (1998) 5417.

[20] T.Blum, A.Sony,Phys.Rev.Lett. 79 (1997).

[21] T.Blum, Talk delivered at LATTICE'98.

[22] S.Aoki, Y.Taniguchi, hep-lat/9711004.

[23] Y.Kikukawa, H.Neuberger, A.Yamada, hep-lat/9712022. 